\title{
NASA's Analog Missions: Driving Exploration Through Innovative Testing
}

\author{
Marcum L. Reagan ${ }^{1}$ and Barbara A. Janoiko ${ }^{2}$ and Michele L. Parker ${ }^{3}$ and James E. Johnson ${ }^{4}$ \\ NASA/Johnson Space Center, Houston, TX 77058 \\ Steven P. Chappell, Ph.D ${ }^{5}$ and Andrew F. Abercromby, Ph.D ${ }^{6}$ \\ Wyle Science, Technology \& Engineering Group, Houston, TX 77058
}

\begin{abstract}
Human exploration beyond low-Earth orbit (LEO) will require a unique collection of advanced, innovative technologies and the precise execution of complex and challenging operational concepts. One tool we in the Analog Missions Project at the National Aeronautics and Space Administration (NASA) utilize to validate exploration system architecture concepts and conduct technology demonstrations, while gaining a deeper understanding of system-wide technical and operational challenges, is our analog missions. Analog missions are multi-disciplinary activities that test multiple features of future spaceflight missions in an integrated fashion to gain a deeper understanding of system-level interactions and integrated operations. These missions frequently occur in remote and extreme environments that are representative in one or more ways to that of future spaceflight destinations. They allow us to test robotics, vehicle prototypes, habitats, communications systems, in-situ resource utilization, and human performance as it relates to these technologies. And they allow us to validate architectural concepts, conduct technology demonstrations, and gain a deeper understanding of system-wide technical and operational challenges needed to support crewed missions beyond LEO. As NASA develops a capability driven architecture for transporting crew to a variety of space environments, including the moon, near-Earth asteroids (NEA), Mars, and other destinations, it will use its analog missions to gather requirements and develop the technologies that are necessary to ensure successful human exploration beyond LEO. Currently, there are four analog mission platforms: Research and Technology Studies (RATS), NASA's Extreme Environment Mission Operations (NEEMO), In-Situ Resource Utilization (ISRU), and International Space Station (ISS) Test bed for Analog Research (ISTAR).
\end{abstract}

\section{History}

P roject Backgrounds: NASA's Apollo program demonstrated analog missions as an effective method for developing extravehicular activities (EVAs), surface transportation and geophysics capabilities for the lunar missions. Following Apollo, much of NASA's analog activities remained dormant until 1997, when the Desert Research and Technology Studies (Desert RATS) mission was formed to re-focus on the technology development of next generation surface exploration space suits at remote testing locations outside Flagstaff, Arizona and other desert locations. Desert RATS grew to include more than just suit technology work and soon evaluated human-robotic interactions with an ever increasing focus on an anticipated return to lunar exploration. As NASA's Constellation program gained prominence, conceptual trade studies moved from meeting rooms to the harsh desert environment

\footnotetext{
${ }_{1}^{1}$ NEEMO Mission Director, Analog Project Office, EA329, AIAA junior member.

${ }^{2}$ Project Manager, Analog Project Office, EA329.

${ }^{3}$ Deputy Project Manager, Analog Project Office, EA329.

${ }^{4}$ Systems Engineering and Integration Lead, Analog Project Office, EA329.

${ }_{6}^{5}$ Research Specialist, Exploration Analogs and Mission Development, Wyle/HAC/37C, and AIAA Senior Member.

${ }^{6}$ Deputy Project Manager, Multi-Mission Space Exploration Vehicle Project, Wyle/HAC/37C, AIAA Senior Member.
} 
where rapidly prototyped hardware was tested and evaluated to iteratively converge upon a mature operational concept of lunar surface exploration and candidate technologies.

In parallel with the Desert RATS mission, the first NASA Extreme Environment Mission Operations (NEEMO) mission got underway in 2001, utilizing an underwater habitat provided by the National Oceanic and Atmospheric Administration (NOAA) off the coast of Key Largo, Florida. The initial focus of NEEMO was predominately on training and preparing future space shuttle and space station crewmembers for operating in an extreme environment from which there was no immediate return. Soon NEEMO missions were focused on integrated operations, taking advantage of the fact that gravity in the undersea environment was a variable, and could easily be adjusted from 0-g (neutrally buoyant) to lunar, martian or any other destination for EVAs. Because it was a fully crewed end-to-end mission, it proved itself a good environment for testing operational concepts and tools as well.

The In-Situ Resource Utilization (ISRU) project was formed in 2008. While it is largely focused on technology development and demonstration, facets of it have synergy with NEEMO and RATS (namely the navigation software, and methodology for performing traverses in search of water.) ISRU missions to date have been conducted in 2008, 2010 and 2012 in partnership with the Canadian Space Agency, the University of Hawaii, and the Pacific International Space Center for Exploration Systems.

The International Space Station (ISS) Test bed for Analog Research (ISTAR) was established in 2010 as the inspace, high-fideltiy operational analog to maximize use of the ISS platform. It will be used to evaluate new exploration technologies, capabilities, and operational concepts to better comprehend and reduce or mitigate risks related to human exploration of NEA or Mars. Some of the more prominent advantages offered by the ISS as an analog are long mission durations ( 6 months), real astronauts as test subjects, and a micro-gravity environment. ${ }^{1}$

Over the years, NASA has participated in other analog missions, such as the Pavilion Lake Research Project (PLRP), and Haughton Mars Project (HMP). PLRP was formed in 2004 to research the discovery of some rare microbialites that exist in Pavilion Lake, British Columbia, Canada. These microbialites offer a unique glimpse into the earliest form of life on Earth, and the techniques developed for exploring them are also transferable to planetary exploration challenges ${ }^{2}$. HMP was formed in 1997 to study the many similarities between the surface of Mars and those found on Devon Island, Canada. A meteorite impact that formed Haughton Crater millions of years ago, combined with the high arctic desert climate, make it in many ways an excellent Martian surface analog.

Organization: In 2011 NASA created the Analog Missions Project under a new Advanced Exploration Systems (AES) program. This project is managed at Johnson Space Center (JSC), but draws on resources from other NASA centers as well. It also has responsibility for collecting and formulating agency wide objectives for analog missions, planning and executing the missions that are conducted each year. The four currently NASA funded analog missions (NEEMO, RATS, ISRU and ISTAR) were placed under the AES Analog Missions Project to take advantage of the synergy and core expertise across them.

A key part of the Analog Missions Project is the Exploration Analogs Mission Development (EAMD) team. ${ }^{3}$ The EAMD was initiated in March 2009, and chartered to support:

- Exploration Analogs by ensuring a rigorous approach and the use of consistent operational products, tools, methods and metrics across all NASA analog activities to enable iterative development, testing, analysis, and validation of evolving exploration ops concepts

- Mission Development by providing detailed EVA and surface ops analysis and developing assembly, maintenance and science tasks for selected exploration architectures

The two elements of EAMD's charter are closely related and allow detailed, informed, iterative development and refinement of exploration architectures, ops concepts, and technology development. This ensures that the required level of rigor and consistency is applied before, during and after analog field tests so that data collected is usable and relevant to NASA's exploration architecture development and technology development priorities.

\section{Benefits of Analog Missions}

Analog missions benefit NASA in several ways, specifically by advancing new exploration capabilities and improving affordability. Ultimately this leads to a better understanding of the real requirements, more rapid development, and more operationally friendly vehicles which also require less sustained operations costs.

Analog missions advance new capabilities in two ways. The first is in maturing operational concepts and activities, which is done by enabling mission concepts to be tested and validated through formalized testing that includes extensive data collection and evaluation. By incorporating questions regarding future operational architectures into a progression of analog missions, operational activities are iterated and matured, such as concepts, tools, team activities, and technical expertise. The second way is by demonstrating and maturing advanced technologies. Analog missions allow the demonstration of rapidly prototyped technologies in remote, extreme, and 
challenging test environments. These technologies are then matured through iterative testing in integrated mission scenarios that enable operationally-influenced design.

Analog missions improve affordability in two ways as well. The first is in improving system design throughout a system's conceptual development and preliminary design by uncovering technical deficiencies and lessons learned in an operational setting prior to flight development. This "build-a-little, test-a-little" approach in analogs allows us to optimize end-user performance for orders of magnitude less cost than traditional methods. The second is by promoting collaborations through innovative partnerships with international and external organizations, and by formulating collaborations between various NASA centers, other government institutions, academia, and private enterprise. Facilitating such partnerships improves collaboration through development of innovative technologies and sharing of logistical resources to reduce operational costs.

\section{NASA Analog Missions in 2012}

The rigorous process for testing objectives and analyzing data for analog missions is critical to building and assessing a capability driven exploration framework. In fiscal year 2012 four analog activities were chosen for funding: RATS, NEEMO, ISRU and ISTAR. RATS and NEEMO were primarily focused on the challenge of conducting exploration operations on a Near Earth Asteroid (NEA). ISRU was focused on the operations and technology needed to identify and ground truth locations with high water (ice) content in lunar soil prior to human arrival, and ISTAR was focused on operational techniques for mitigating long comm delays (on the order of those that would be seen for a Mars mission) and on-board procedures that promote crew autonomy.

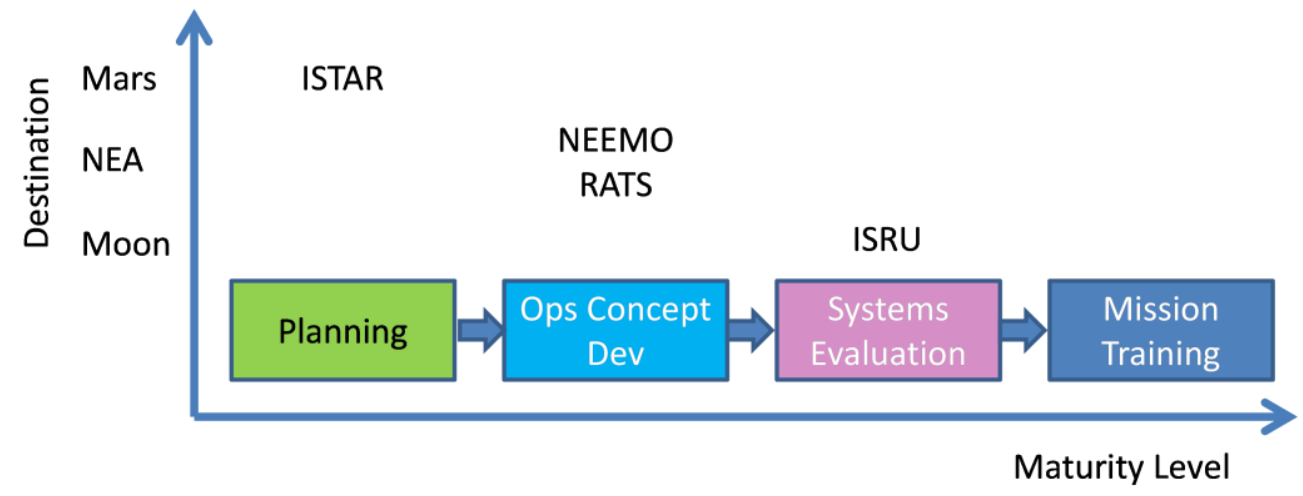

Figure 1. Analog Maturity and Destinations

Analog missions might be thought of relative to their maturity levels, as well as destinations. At the more immature, or Planning end of the spectrum are those missions that are really just in the planning stages (see Fig. 1. Analog Maturity and Desinations.) Progressively maturity increases to the Ops Concept Development stage. At this point ops concepts are being devised and matured, in a cycle that is rapidly feeding hardware requirements. Next is the Systems Evaluation stage. Hardware and systems are mature enough to be tested in an end-to-end way, and the previously developed concepts are being fine tuned and proven. Finally, in the Training stage both hardware and ops concepts are mature enough to use for both crew and mission controller training scenarios.

As Fig. 1 shows, ISTAR is still largely in the planning stage, NEEMO and RATS are in the Ops Concept Development stage, and ISRU is in the Hardware Evaluation stage. The Mission Training stage would occur in the year or so immediately prior to the mission, once actual crews were assigned, the destination was well known, and the mission design was fairly mature.

Much of NASA's focus on analog missions occurs in the Ops Concept Development stage, because we can rapidly prototype multiple different scenarios and quickly get a sense for what will work best and what architectural capabilities will be needed to support the mission concept. This allows us to hone our ops concept, which in turn helps hone vehicle requirements in a relatively short time frame, and for a relatively low cost. The NEEMO and RATS mission will be explained in greater detail later to illustrate how that is done.

\section{Near Earth Asteroid (NEA) Exploration}

Since both RATS and NEEMO in 2012 were focused on NEA exploration questions and techniques, a short introduction to the subject is in order. 
NEAs are objects that come within 45 million $\mathrm{km}$ of Earth orbit and can range in size from a few meters in diameter to more than $30 \mathrm{~km}$ across as in the case of asteroid (433) Eros. NEAs appear to have a range of compositions and structures based on evidence obtained via ground-based observations, robotic spacecraft missions such as Hayabusa and laboratory analyses of meteorites. They also represent the oldest rocks in the solar system and contain clues to the development and formation of all the planets, the Earth, and the moon. The cratering record from both the Earth and the moon indicates that NEAs have impacted the Earth-moon system for billions of years and it is now commonly recognized that the impact of a $10 \mathrm{~km}$ object into the Yucatán peninsula approximately 65 million years ago was the cause of the massive K-T (i.e., dinosaur) extinction event. Estimates suggest that 20,500 +/- 3000 NEAs equal to or greater than $100 \mathrm{~m}$ in diameter exist within our Solar System of which approximately $1 / 5$ th are thought to be potentially hazardous. ${ }^{4-8}$

Exploration Systems: Fundamental to the development of a Capability Driven Framework is identifying the exploration systems that are required for the range of destinations being considered and finding safe, affordable, and effective ways to develop and operate those systems. Design reference missions (DRMs) currently being considered by NASA for human exploration of NEAs include stays in the proximity of the target NEA of between 14 and 56 days during which time the Earth-NEA transit vehicle, Deep space habitat (DSH), and Multi-Purpose Crew Vehicle (MPCV, used for crew launch and re-entry) would remain between 500 meters and $2 \mathrm{~km}$ from the NEA to minimize the possibility of collision (Fig. 2). Exploration and sampling of the NEA surface would be conducted by crewmembers leaving the DSH either in EVA suits with appropriately sized mobility systems to enable brief sorties to the NEA surface or else using Multi-Mission Space Exploration Vehicles (MMSEVs, Fig. 3) with rapid EVA capability to enable multi-day exploration

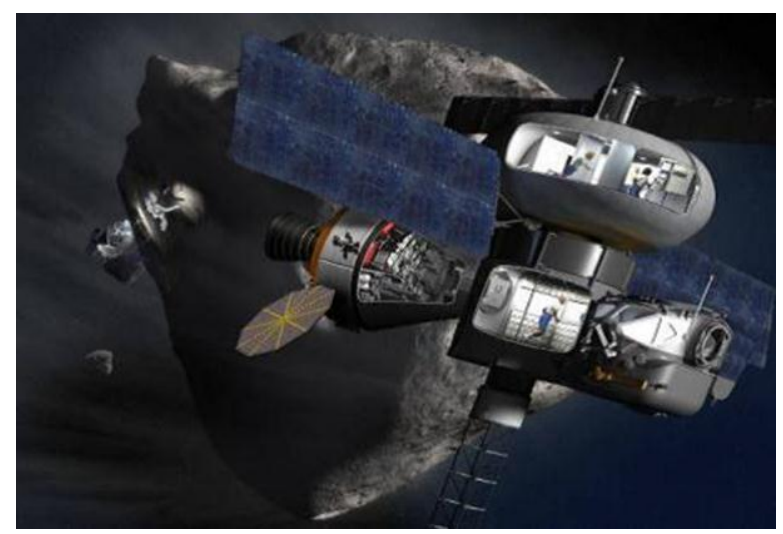

Figure 2: DSH with one MMSEV remaining attached (right), while a second explores at NEA surface (left). MPCV also shown (center). sorties.

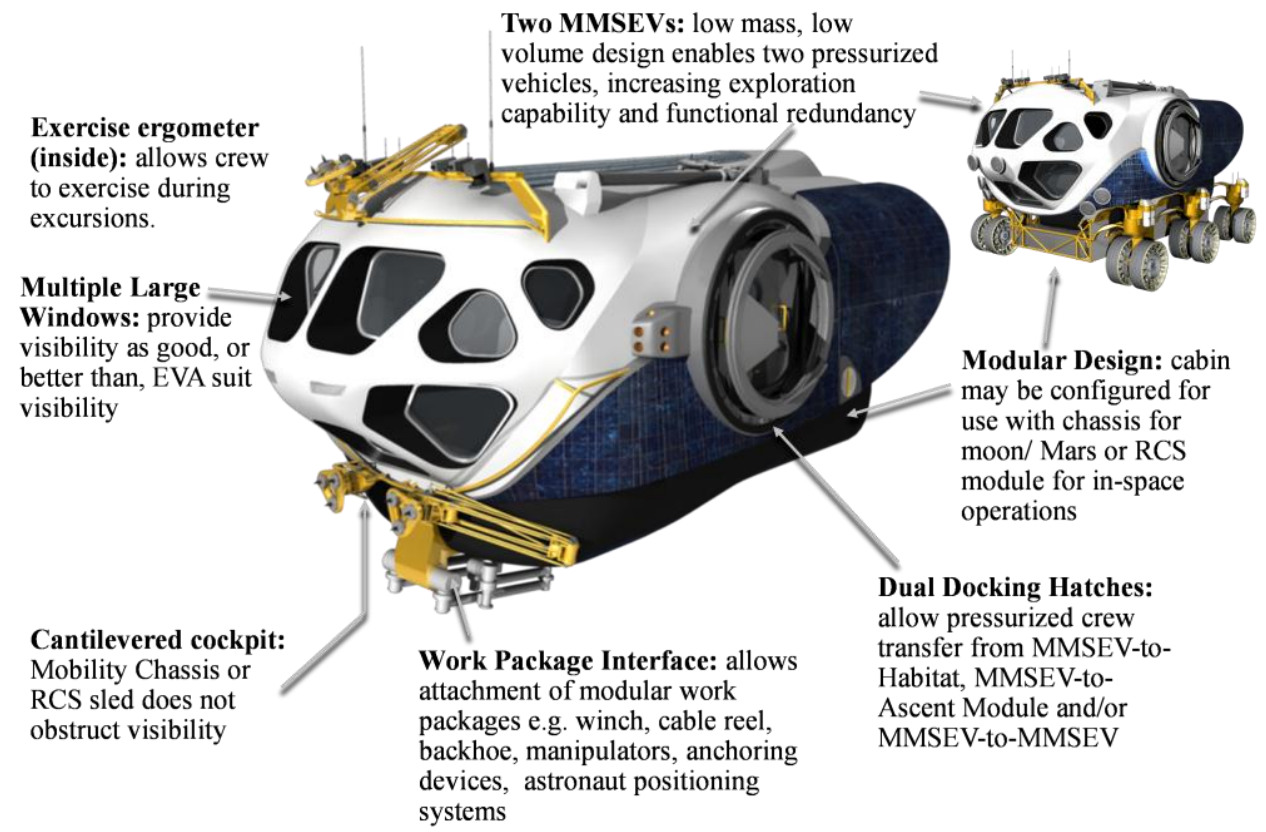

Figure 3. Multi-Mission Space Exploration Vehicle (MMSEV) 
Exploration Techniques: Prior to the human mission would be a robotic precursor mission, which would have collected detailed remote sensing data on the asteroid. This would allow scientists to plan traverses across the asteroid body from one area of high scientific interest to another (represented by the groupings of X's in the enclosed circles in Fig. 4. It also allows for high definition imagery in the simulations used to train the crew to fly the traverses. Finally, the crewed mission would arrive and would perform close observations and sample collections.

The crewed mission has three primary questions that need to be answered: 1) how does one get to a geological site of interest on the asteroid, stabilize themselves sufficiently to do meaningful work there (collect samples and deploy scientific instruments), then translate to the next site and repeat?,2) given the

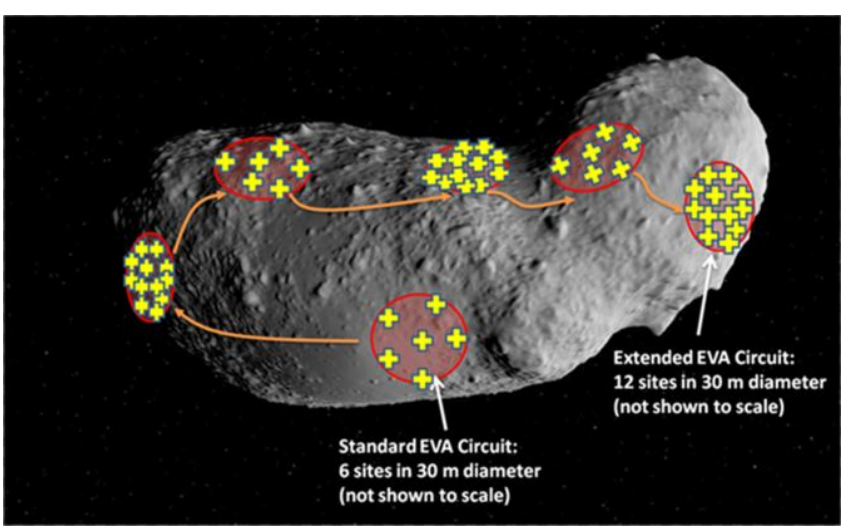

Figure 4: Example of a traverse plan (not to scale) consisting of standard EVA circuits as used during NEEMO and RATS testing. assets at hand (DSH, MMSEV(s), jetpacks), where should the crew members be located and how many MMSEVs should be used to maximize the science collection?, and 3) how much of a factor is the communications delay, and how can that best be mitigated? The 2012 NEEMO and RATS missions were designed to answer those three questions assuming a crew size of four and a 50-second one way time delay.

Translation and Work: While NASA has extensive EVA experience working with tools in microgravity, as well as some (Apollo) EVA experience in planetary surface exploration, the unique challenge of a NEA is the combination of both. Translating from one geologically interesting site to another, and stabilizing oneself to use tools and take samples from a wide variety of surface compositions in microgravity is a very complicated proposition which is requiring us to create new techniques and tools.

We have two basic approaches we are investigating. The first involves somehow physically anchoring the MMSEV to the NEA. The crew would then perform exploration tasks by going out on EVA and translating over the surface using a boom, translation lines, "EVA jetpacks", or some combination of them. We call these different techniques "anchored" modes, as seen in Fig.

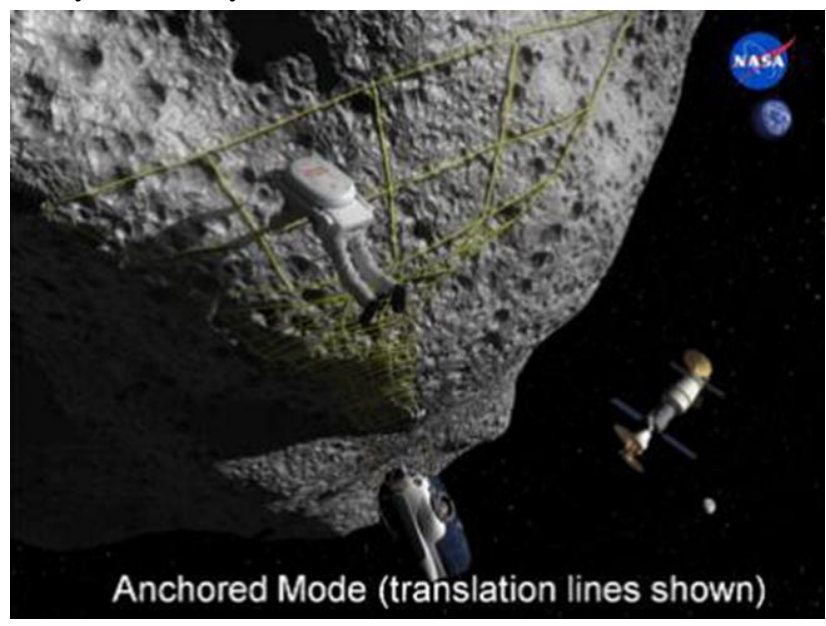

Figure 5. Example Anchored Mode 5.

The other involves staying attached to the MMSEV via an Astronaut Positioning System (APS) arm that would provide a stable work platform, much as the robotic arms are frequently used for EVAs on the ISS (and Shuttle before that.) Or, the astronaut might be in the vicinity of the MMSEV on an EVA jetpack. Because the MMSEV would be flying around with this crewmember attached, we call these "free-flying" modes, as seen in Fig. 6.

Deployment of Assets: Multiple

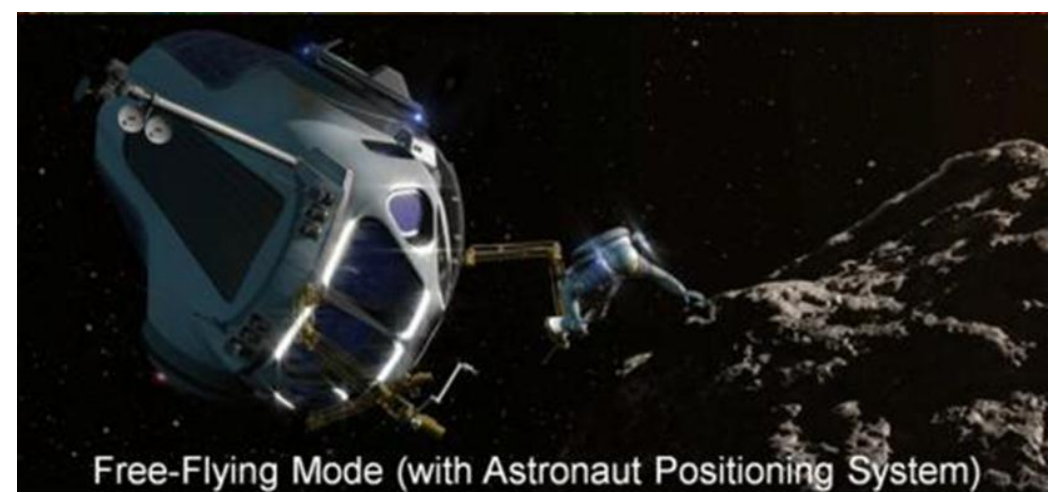

Figure 6. Example Free-Flying Mode possibilities for deploying assets at the NEA have been investigated, and productivity of work assessed. We identify distinct possibilities as "conditions" . Previous analog missions and analysis have narrowed the possibilities for maximum productivity of a crewed mission to two conditions (6 and 7). ${ }^{9,10}$ Condition 6 has some slight variations 
of interest, which we call 6A, 6B, and 6C. These can be seen in Figure 7. The primary variables under consideration are how many people remain back in the DSH, how many are required to pilot the MMSEV, how many MMSEVs are optimal, and how many people would be doing an EVA simultaneously.
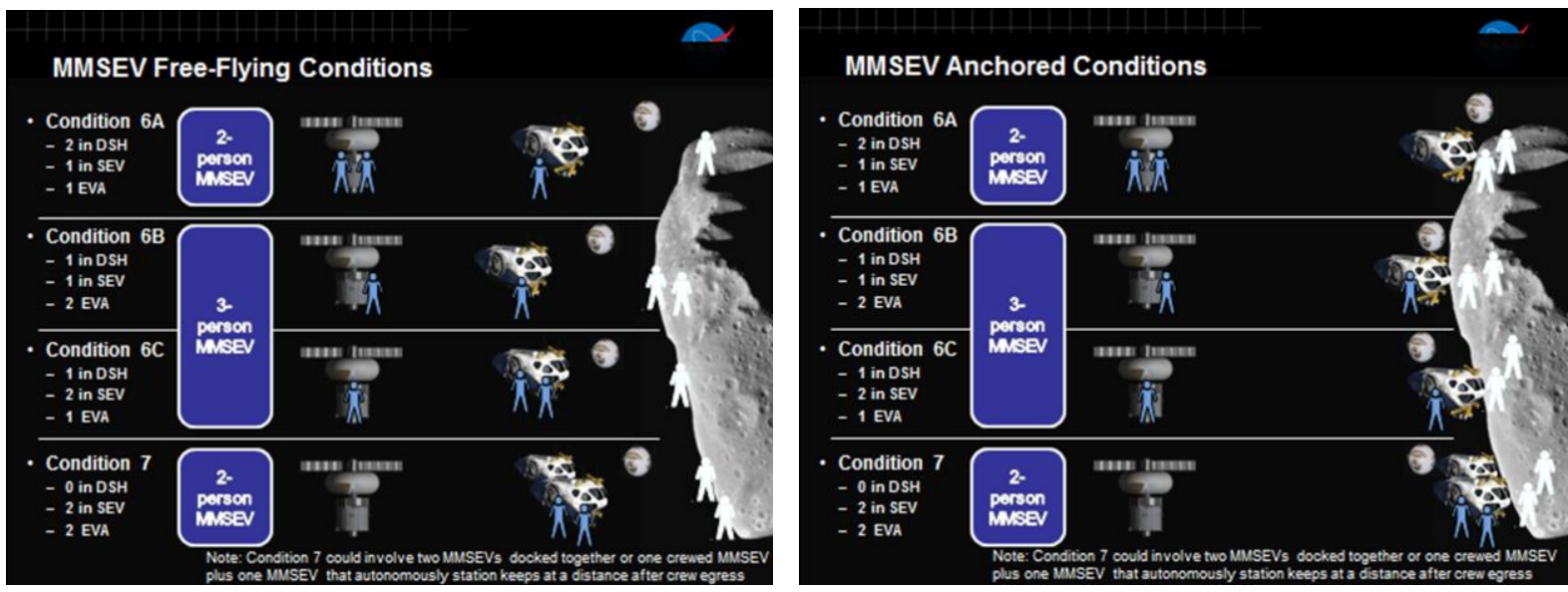

Figure 7. MMSEV Conditions under investigation

Communications Delays: Given the background objective of exploring a NEA, an important consideration to take into account is the communication latency present between the local assets (e.g., the DSH, the MMSEV(s), EV crewmembers) and the earth. Though distances vary based on the relative positions of the Earth and NEA in their orbits, a one-way comm latency of 50 seconds was chosen for NEEMO 16 and RATS 2012, which is representative of a NEA approximately 0.1 astronomical units (AU) from Earth. While unmanned spacecraft have been operated successfully with much greater latencies than this, the largest latency previously for a human mission was on the Apollo missions to the moon, which had a one-way delay of less than 2 seconds. Communications for human missions have multiple components, all of which are impacted by the delay and have to be considered: voice, text, streaming video, video conferencing, data, command, file transfers, and internet use.

\section{NASA Extreme Environment Mission Operations (NEEMO)}

The first 2012 mission conducted was NEEMO 16. This 12 day mission occurred June $11-22,2012$ and derived its name from being the $16^{\text {th }}$ NEEMO mission to date. It built upon the work started during the NEEMO 15 mission. ${ }^{11}$ The mission was focused on exploration of a NEA, and addressed two key architectural questions:

1. What combination of systems (MMSEV(s), DSH, EVA, robotic systems, sample selection and curation, communication and navigation, etc.) is most effective for human exploration of a NEA?

2. How does communications delay affect productivity during human exploration of a NEA, and what tools and techniques would best mitigate the effects of the delay?

NEEMO Infrastructure: The Aquarius Reef Base is a unique ocean science and diving facility. It includes Aquarius (Fig. 8), the only operational undersea research habitat in the world, and a shore based field station. It is owned by the National Oceanic and Atmospheric Administration (NOAA), and operated out of Key Largo, Florida by the University of North Carolina at Wilmington. It is highly sophisticated in its logistical infrastructure, and has not required major modifications to support unique NASA needs. It has supported over 100 saturation missions to date.

Aquarius is similar in size to the U.S. Laboratory module on the International Space Station ( $15 \mathrm{~m}$ long X $4.5 \mathrm{~m}$ in diameter). It is firmly secured to a sand patch surrounded by large spur and groove coral reefs on three sides. It sits in water 60 feet deep, but the entrance level is actually closer to 50 feet, which corresponds to an internal pressure of $\sim 2.5$ atmospheres. At this depth, aquanauts living and working in the habitat become exposed to excessive levels of nitrogen within the first few hours and must commit to staying in the habitat and undergoing a decompression schedule before returning to the surface. This type of diving is called

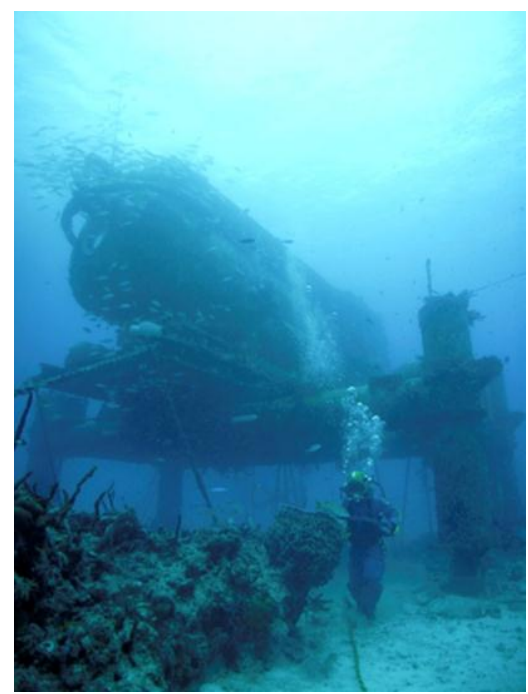

Figure 8. Aquarius habitat

American Institute of Aeronautics and Astronautics 
"saturation" diving, referring to the complete saturation of the body tissues by nitrogen. A diver in this condition will quickly experience the onset of the "bends" if he returns to the surface without going through the requisite decompression schedule, and would most likely experience injury and even death if not treated. The danger is real and the environment is truly extreme, which is one of the key reasons it makes such a good analog to living in space. Aquanauts participating in these missions must utilize their training, skills, knowledge and teamwork to ensure their safety and mission success.

Permanently anchored above Aquarius is a $10 \mathrm{~m}$ Life Support Buoy, or LSB (Fig. 9). Onboard the LSB are redundant generators and compressors which provide electrical power and fresh air via umbilical to the habitat. Separate umbilicals provide

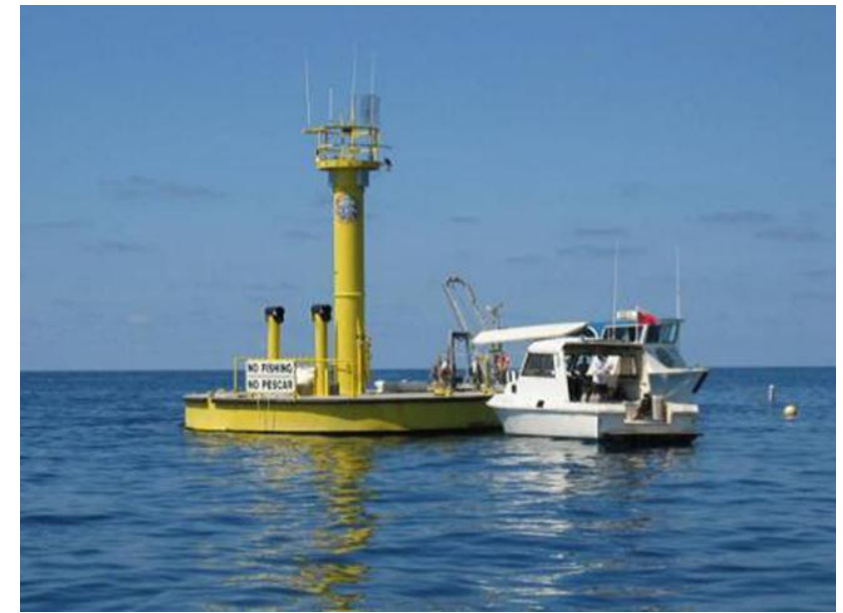

Figure 9. Aquarius Life Support Buoy communications connectivity. From the LSB the signal is relayed via microwave to the ARB headquarters in Key Largo. This allows Aquarius to have real-time voice communication (radio and telephone) and internet connectivity. It also allows the "watch desk" at ARB to monitor video and systems telemetry real-time, which they do 24/7 during a mission.

In addition, NASA deployed a Mobile Mission Control Center (MMCC, Fig. 10, 11) which was able to comfortably seat 20 operators.

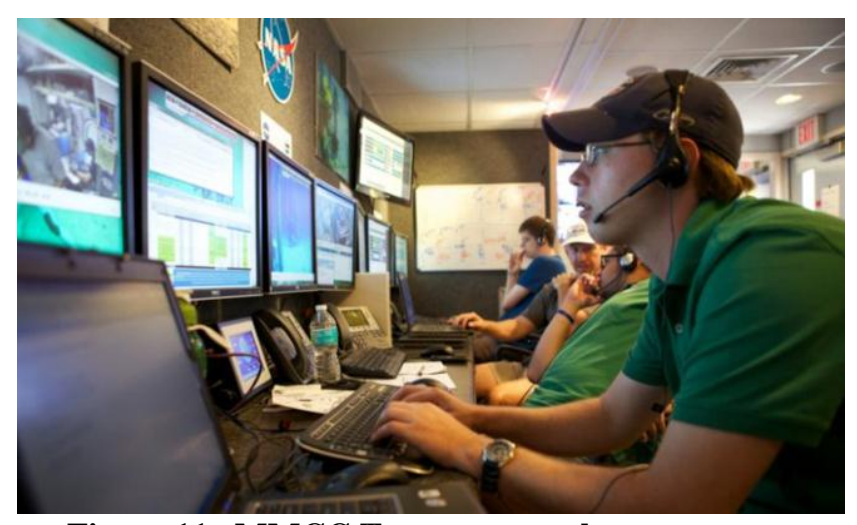

Figure 11. MMCC Team on console

The last major asset in place to support the mission was a pair of Deepworker 2000 mini-submersibles (Fig. 12). These played the role of the MMSEV. They had full communications capability with each other, the extravehicular $(\mathrm{EV}) \mathrm{crew}$, the habitat (playing the role of the DSH) and the MMCC during operations. They were outfitted with attachments appropriate to the task being simulated (e.g., an Astronaut Positioning System, and a stinger used to secure it to the asteroid surface.)

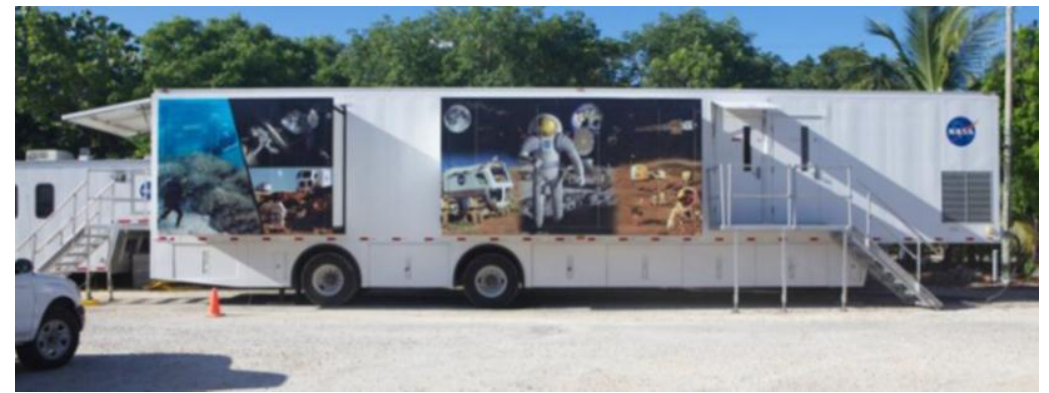

Figure 10. Mobile Mission Control Center (MMCC)

Positions supported included Mission Director, EVA Officer, Capcom (primary communicator with the crew), HRP (Human Research Program), Planning, Flight Surgeon, Data, Communications, Educational and Public Outreach, Imagery, and Science. The MMCC team was responsible for the daily plan and support products (e.g., procedures, data sheets, etc.) and overall priorities and direction for the mission. The MMCC was staffed 14 hrs per day.

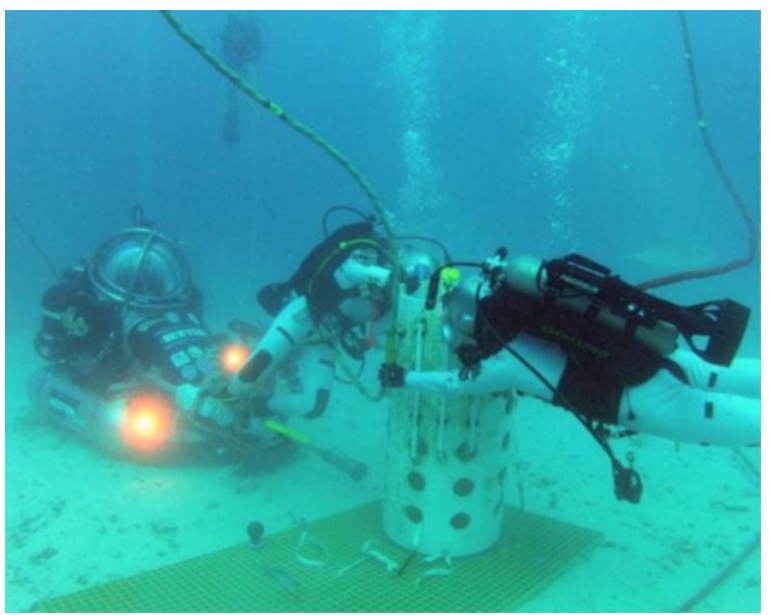

Figure 12. Deepworker submersible outfitted with an Astronaut Positioning System and stinger 
In order to safely deploy and recover the submersibles, the MV Liberty Star (Fig. 13) was brought in. The Liberty Star had a central role in recovering Space Shuttle Solid Rocket Boosters since the beginning of the Space Shuttle Program. In addition to being a stable ship with dynamic positioning thrusters, it has a large hydraulic crane, experienced crane operators, and a very experienced team of divers and provided the perfect platform for supporting the submersible operations.

NEEMO 16 Mission: The NEEMO 16 mission was a 12 day mission with 6 crew members. The four NASA affiliated crewmembers responsible for performing the NEA exploration tasks were: NASA astronaut Dottie Metcalf-Lindenberger (crew commander), Japanese Aerospace Exploration Agency (JAXA) astronaut Kimiya

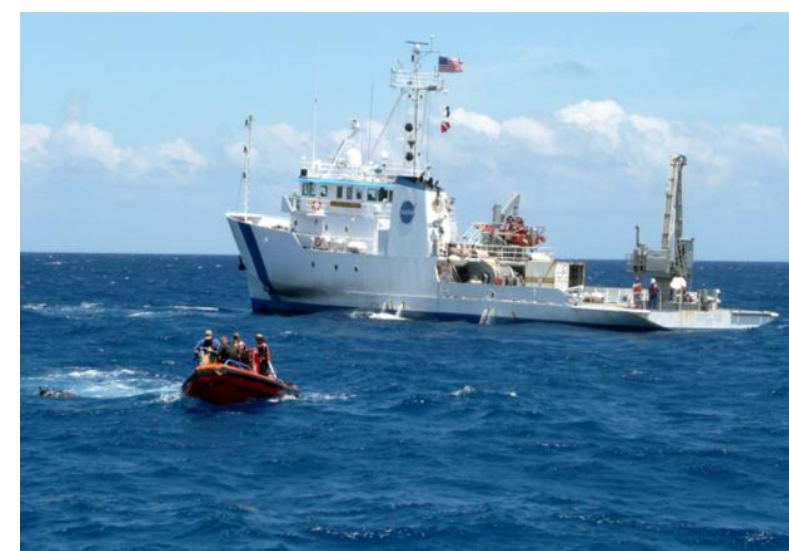

Figure 13. MV Liberty Star Yui, European Space Agency (ESA) astronaut Tim Peake, and Dr. Steve Squyres from Cornell University. Professional aquanauts James Talacek and Justin Brown from the Aquarius Reef Base (ARB) rounded out the crew.

The mission was designed to complete 3 days of Anchored Mode tasks, 4 days of Free Flying Mode tasks, and 1 day with the freedom to utilize the best combination of techniques seen. Additional objectives included HRP investigations into habitability tools and crew teamwork and autonomy during high criticality, high novelty situations with a comm delay. The entire mission was conducted under a 50 second (one way) comm delay, with some targeted exceptions (e.g., educational outreach events.)

A circuit of representative NEA EVA tasks was created on the seafloor near the Aquarius habitat. Each of the four NASA crewmembers completed the NEA Circuit every day (sometimes multiple times per day) under each of the different test Conditions and modes. For example, 2 crewmembers might be paired on a given day for a morning EVA of 3 hours, during which they might investigate anchored modes utilizing a small boom. One of the crewmembers remaining in the habitat would play the role of the "quarterback" of the EVA (which we term "IV", meaning the intra-vehicular crewmember), and the $4^{\text {th }}$ would help with the IV role until the EVA was well established, and then would typically have educational or public outreach activities to support.

The purpose was to assess procedures and equipment for collecting and documenting different types of samples on a NEA surface. The IV crewmember was heavily engaged in the EVA, and actively collecting data from each crewmember on the acceptability of each task in that configuration, the simulation quality, and an assessment of the overall capability. In the afternoon the two pairs would swap roles. At various points in the mission the crew would discuss and reach consensus on the ratings to give for each task and mode. In this way we collected over 1200 data points on the various capabilities.

The tasks on the circuit were

- Float sampling

- $\quad$ Rock chip sampling

- Soil sampling

- Core drill sampling

- Geophysical array deployment (a simulated complex instrument array)

- Orbital Replacement Unit (ORU) deploy (representative of a instrument or piece of equipment)

In order to get from site to site to collect these samples and perform these activities, various translation and worksite stabilization modes were also investigated. For the MMSEV Anchored modes these included

- Translation lines

- A small boom

- EVA jetpack

Some representative pictures of these activities being accomplished follow in Fig. 14-17: 


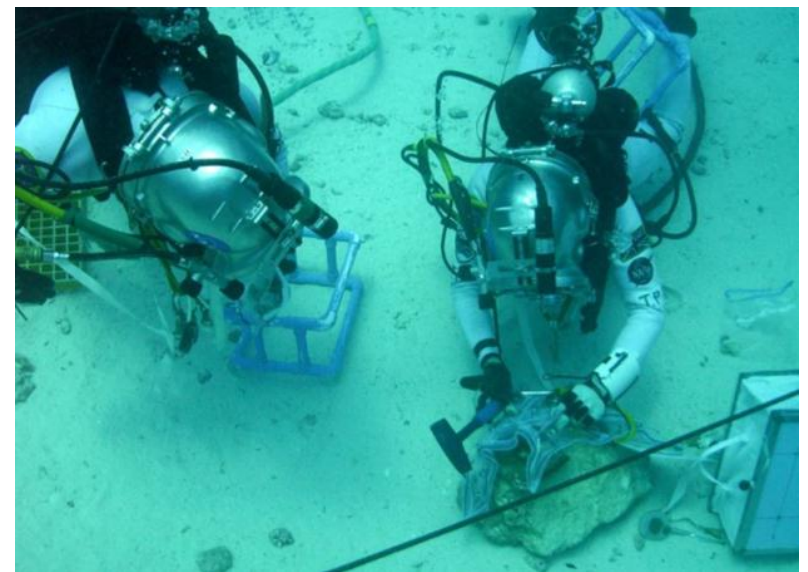

Figure 14: Rock Chip Sampling, anchored mode

Comm Delay: Using a comm latency emulator and rules of engagement, we were able to operate a substantial portion of the NEEMO 16 mission with a baseline comm latency affecting the wide range of communications involved in a crewed mission: voice, text, streaming video, video conferencing, file transfer and internet use.

For normal operational comm we used both voice and text for 2-way communications between the MMCC and the crew. For simulated Private Medical Conferences we used 2-way Videocon or 2-way voice. Although most of the Public Affairs Office (PAO) and educational outreach events were conducted in real time, we did do three PAO events (using 2-way videoconferencing) with the 50-second comm delay in place to look at various techniques for ending up with a final product that would be publicly digestible.

Internet usage was not time delayed. As a practical matter one could not "surf" the internet over a time delay this long as web pages would time out. What would be possible, however, is to crawl over a discrete site and package it to some number of links deep, and then deliver that as a file. As long as the user didn't go to links deeper than had been packaged, it would have the feel of browsing that one site. The way we approached this was to use a "rule of engagement" that any discrete site could be browsed provided the MMCC was notified 2 hours in advance. In this way we simulated that a site could be thus packaged and that file put onboard.

We also did several Just in Time Training (JITT) tasks that each crewmember participated in. One of the significant challenges that will arise for distant exploration missions is how to deliver highly detailed training to the crew quickly. We know that prior to a long-duration mission, it is impossible to think of and train the crew for every contingency. Additionally, the mission itself will last so long that even the things we did train the crew for initially might be forgotten. Because of the time-delay induced communication issues, the current proven method of the Mission Control Center guiding astronauts through each step of the problem is not a good solution to time-critical activities. So how does an astronaut dig into the guts of - and fix- something that they either never had training on in the first place or, received training on but it was so long ago that they do not remember? 
Since our NEEMO aquanauts did not have any training on Aquarius - the professional ARB aquanauts on the crew are responsible for the habitat - Aquarius maintenance activities offered an excellent way to investigate this question. During our NEEMO 16 mission, we planned several complex, maintenance-type tasks on Aquarius for our aquanauts to perform using JITT. We provided the crew with training products that were simple enough to have been developed overnight and that used a mobile tablet device for viewing. The aquanauts used these products to successfully train themselves and carry out these unfamiliar tasks.

The NEEMO 16 mission was highly successful. All major mission objectives were accomplished and over 1200 data points were collected to inform our decisions on NEA exploration operational concepts, EVA tools, and communications delay mitigation tools and techniques.

\section{Research and Technology Studies (RATS)}

The RATS 2012 mission is scheduled for August 20 - 29, 2012 and will utilize various capabilitlies located at NASA/JSC. This 10 day mission builds on previous RATS and NEEMO missions, ${ }^{9-12}$ and will also be focused on exploration of a NEA and will utilize 5 crewmembers. The RATS 2012 crew includes two experts from NASA's EVA community, David Coan and Allison Bolinger, two geologists, Trevor Graff and Elizabeth Rampe, and a member of the Analog Missions Project office, Marc Reagan. The mission will also use a 50-second one-way communications latency. The same types of data, using the same techniques as described for NEEMO 16 will be used. However, RATS and NEEMO missions, having different inherent strengths and weaknesses, provide different perspectives on the NEA exploration problem. Where NEEMO 16 allowed for a very high fidelity assessment of EVA tools and the actual interface between the astronaut and the asteroid surface, RATS 2012 has much higher fidelity in several other significant areas discussed later in this report.

The mission will address the architectural questions that were addressed on the NEEMO mission, and additionally:

1. How does crew workload, productivity, and propellant usage vary as a function of crew distribution, exploration ops concept, and NEA surface velocity and centripetal acceleration?

2. Are Generation (Gen) 2A MMSEV cabin human factors acceptable?

RATS Infrastructure: A number of assets will be involved in the RATS 2012 test, including the Analog Mission Control Center (AMCC), Active Response Gravity Offload System (ARGOS), virtual-reality laboratory (VR Lab), and a high-fidelity NEA simulation which uses advanced modeling and graphics to assess the dynamics of NEA proximity operations with an MMSEV. It will also include the Gen 2A MMSEV prototype cabin with suit ports and a Mark III Mock-up suit. A Deep Space Hab (DSH) work station will be located near the test area for use by the assigned DSH IV crew.

The Gen 2A MMSEV (Fig. 18) is the third prototype vehicle developed by the MMSEV project and is the first in the second generation. Generation 2 is an intermediate step between the Gen 1 concept development vehicles that enabled rapid and cost effective design, build, and test of different concepts, and the Gen 3 protoflight vehicles that will be designed and built as protoflight vehicles capable of being flown in space. Preliminary habitability and human factors testing of the Gen 2A MMSEV was conducted during December 2011 and January 2012. During RATS 2012 testing two pairs of crewmembers will live continuously for 3 days and nights in the MMSEV and will provide additional crewmember data on all aspects of the MMSEV while being operated within a high fidelity operational simulation.

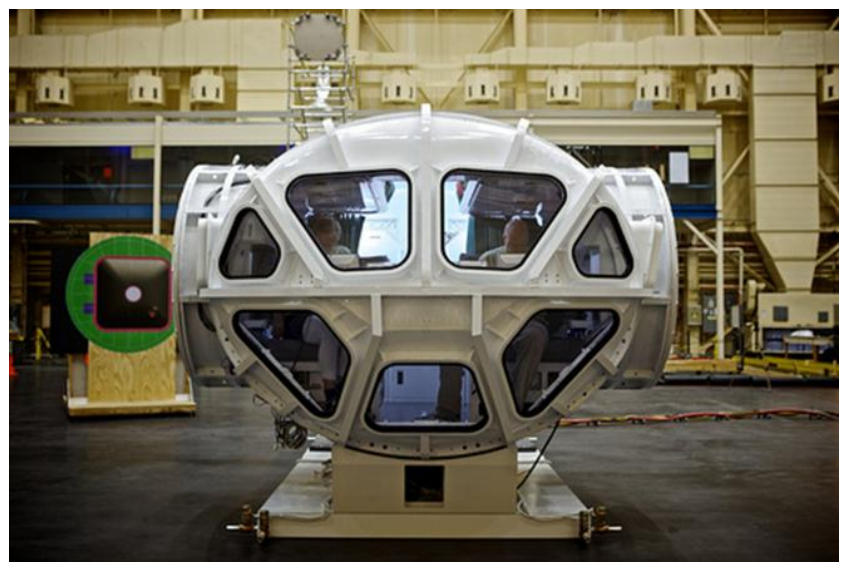

Figure 18. Generation 2A MMSEV Cabin 
The Gen 2A MMSEV will be located within the JSC Building 9 high-bay throughout the RATS 2012 test and will be surrounded by high-resolution video walls (Fig. 19) on which video generated by the MMSEV NEA Simulation will be displayed. Crewmembers inside the MMSEV will use the displays, controls, and views of the video walls through the windows to operate the MMSEV within the immersive NEA simulation environment throughout the ten-day test (Fig. 20).

The MMSEV Near Earth Asteroid (NEA) simulation supports the flight phase of the MMSEV with operation in proximity of a NEA, specifically Itokawa, with high-fidelity physics based assessment of design, and Human-In-The-Loop (HITL) evaluation. The NEA simulation uses the GN\&C "Baseline 1" assumptions from the MMSEV Reaction Control System (RCS) sled flight concept team which includes jet layout, thruster characteristics, and mass properties.

Crewmembers will ingress and egress the Gen 2A MMSEV via functional suitports into unpressurized mockup EVA suits (Fig. 21) to enable initial evaluation of the suitport human factors and to maximize the fidelity of the operational simulation. After completing an egress/ingress cycle, crewmembers will move to either the VR Lab or ARGOS facility to conduct the EVA activities.

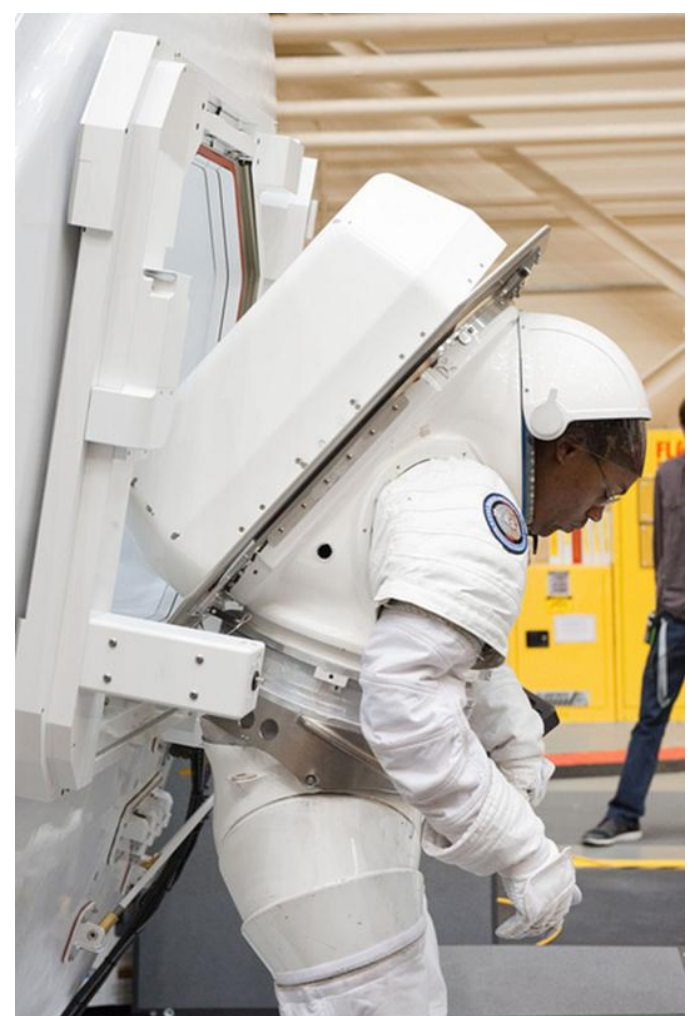

Figure 21. Mockup space suit on a suit port

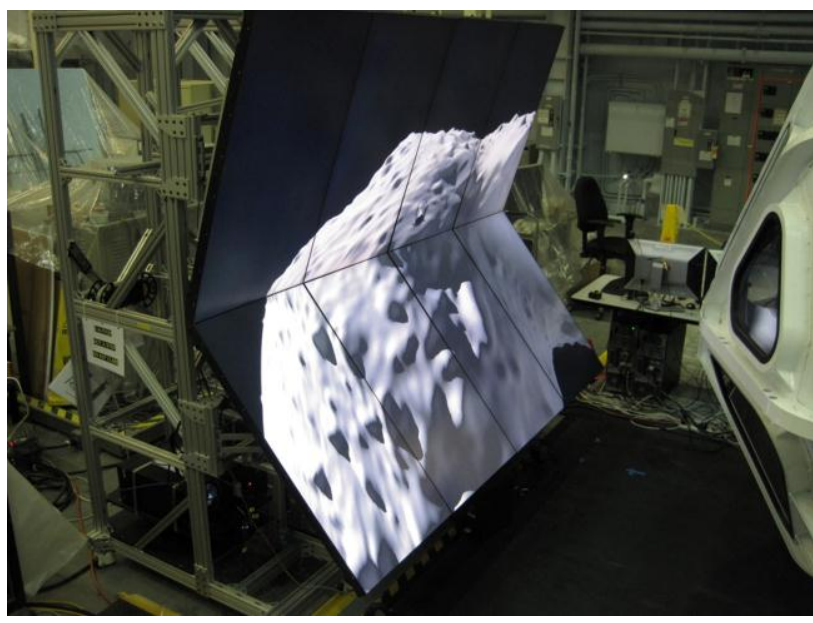

Figure 19. NEA Simulation Video Wall

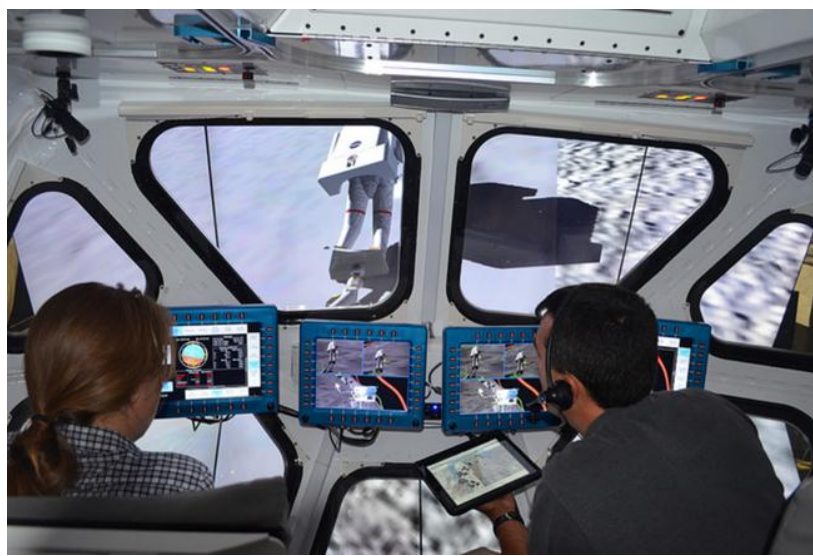

Figure 20. NEA simulation from inside the Gen 2A cabin

ARGOS (Fig. 22) provides the ability to precisely offload test subjects to simulate weightlessness. Within the ARGOS test area pallets of simulants as well as large boulders have been positioned to enable testing of the EVA circuit and tools. ARGOS will be used in two ways during RATS 2012. It will be used as part of the integrated simulation including the MMSEV, NEA Sim, VR Lab, DSH workstation, and AMCC and will also be used to conduct standalone testing outside of the larger simulation. When operating within the larger simulation, crewmembers will have voice communications with other

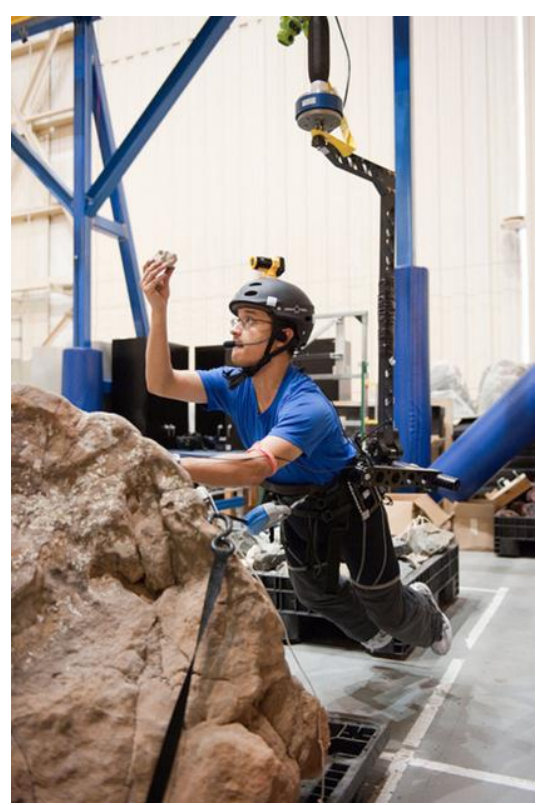

Figure 22. Active Response Gravity Offload System (ARGOS) 
crewmembers and AMCC. The other crewmembers and AMCC will also be able to view video from a helmet camera worn by the ARGOS crewmember as well as a situational camera view showing the crewmember within the ARGOS circuit area.

The MMSEV NEA simulation is integrated with the VR Lab to enable the MMSEV to be flown with up to two EVA crewmembers operating with EVA Jetpacks and/or operating from a single-person foot restraint (Astronaut Positioning System, APS) attached to the front of the MMSEV. Helmet camera views from EVA crew in the VR Lab (Fig. 23) will be viewable by MMSEV crewmembers as well as at the DSH workstation and, with communications latency, in the AMCC. EVA crewmembers being controlled from the VR Lab will also be visible in MMSEV camera views as well as in video wall views viewable through MMSEV windows. The VR Lab will be used by all EVA crewmembers during Free-Flying test conditions and by one of the two EVA crewmembers during Anchored test conditions.

Several test conditions involve one or two crewmembers supporting exploration operations from a workstation that would be located inside the Deep Space Habitat, which would be located approximately 1-2 km from the MMSEV and NEA surface. Crewmembers at the DSH workstation will have access to voice, text, and video communications from EVA crewmembers, MMSEV crewmembers, as well as AMCC. Nominally, DSH crewmembers will serve as Capcom for the MMSEV and EVA

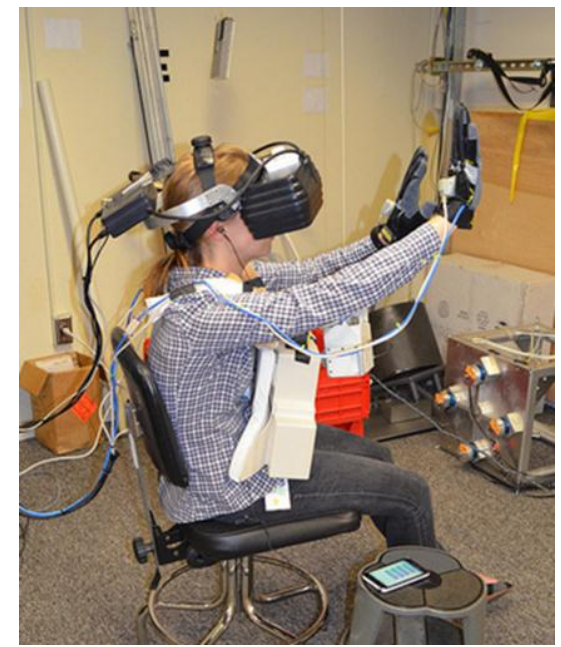

Figure 23. Active Response Gravity Offload System (ARGOS) crewmembers and will be the primary, perhaps only, crewmember(s) receiving communications directly from AMCC. Previous analog field testing at Pavilion Lake, Desert RATS, and NEEMO has shown this to be an effective way of mitigating the effects of communications latency by ensuring that communications from AMCC do not interrupt EVA crewmembers or MMSEV pilots at inopportune times. Instead, the IV Capcom, who has negligible communications latency with the MMSEV and EVA crew, coordinates the activities and passes along information from AMCC as appropriate. In one test condition, the IV Capcom role during EVAs will be performed by a second crewmember inside the MMSEV instead of from the DSH.

The Analog Mission Control Center (AMCC) is a one room facility located at the Johnson Space Center established to allow the monitoring and coordination of test activities and crews within JSC as well as at remote locations. The AMCC is staffed by science and support teams working at consoles located across the hall from the International Space Station Flight Control Room. It provides the capability to manage two way communications with flight crews and assets via audio, video, data and text exchanges for efficient exploration operations.

\section{International Space Station (ISS) Test bed for Analog Research (ISTAR)}

The 2011 NASA Strategic Plan" ${ }^{13}$ includes a sub-goal (1.1) to "Sustain the operation and full use of the International Space Station (ISS) and expand efforts to utilize the ISS (Fig. 24) as a National Laboratory for scientific, technological, diplomatic, and educational purposes and for supporting future objectives in human space exploration." In pursuit of that goal, NASA has committed to utilize the ISS for its unique capabilities as an analog to exploration space flight.

The ISS brings several unique capabilities to bear as an analog:

- Micro-gravity environment

- Long duration mission length

- Astronauts as subjects

- Complex operations, procedures and systems

- Extensive ground based support facilities (e.g., the Space Station Training Facility)

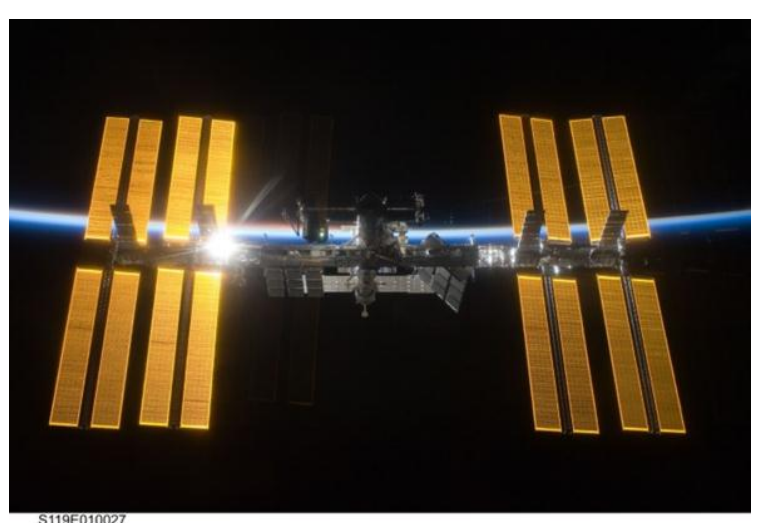

Figure 24. The International Space Station

The ISS can be used to answer exploration questions that require these capabilities.

ISTAR is focused on a simulated Mars transit mission, and envisions a phased approach to reduce exploration risks, answer architectural questions, and execute long-duration exploration mission simulations. This 5-year 
strategic plan ${ }^{14}$ starts by testing risk-mitigating technologies and operational tools, establishing baselines for crew performance, behavior, and medical procedures, and developing and testing countermeasures. It recommends simulations with increasing periods of flight-crew and vehicle autonomy. The plan is broken into four phases, shown in Figure 25.

\begin{tabular}{|c|c|}
\hline Phase & Major features of plan \\
\hline $\begin{array}{l}\text { A } \\
\text { Evaluate ISS } \\
\text { capabilities } \\
{[2011-2012]}\end{array}$ & $\begin{array}{l}\text { - Assess current ISS operations and activities for a Mars Mission } \\
\text { - Begin to introduce Mars specific operational changes }\end{array}$ \\
\hline $\begin{array}{l}\text { B } \\
\text { Short-period } \\
\text { Simulations } \\
{[2013-2014]}\end{array}$ & $\begin{array}{l}\text { - Incorporate discrete Mars-forward activities } \\
\text { - Increase crew and ground mission autonomy } \\
\text { - Include communications delays typical of Mars missions } \\
\text { - Incorporate exploration related system experiments } \\
\text { - Add "exploration" tasks to post-landing timeline }\end{array}$ \\
\hline $\begin{array}{l}\text { C } \\
\text { Longer-period } \\
\text { simulations } \\
{[2014-2015]}\end{array}$ & $\begin{array}{l}\text { - More rigorous, longer periods of autonomy } \\
\text { - Incorporate more exploration related system experiments } \\
\text { - MCC oversight modified for more autonomy while still protecting ISS } \\
\text { - Post-landing multi-day exploration analogs }\end{array}$ \\
\hline $\begin{array}{l}\text { D } \\
\text { 6-month mission } \\
\text { and crew } \\
\text { deconditioning } \\
\text { [post 2015] }\end{array}$ & $\begin{array}{l}\text { - Transits to Mars (or NEAs) simulated as rigorously as feasible } \\
\text { - Progressively increase communication delays to mimic Mars approach } \\
\text { - On-board science to be compatible with Mars-like mission parameters } \\
\text { - Expanded post-landing exploration mission analogs } \\
\text { - Re-launch crew and begin Return to Earth simulation }\end{array}$ \\
\hline
\end{tabular}

\section{Figure 25. ISTAR Five-Year Strategic Plan}

Though largely in the planning stage, some ISTAR objectives related to operating with long communications delays and increased crew autonomy have already been formulated, approved, and demonstrated to date.

\section{In-situ Resource Utilization (ISRU)}

The $3^{\text {rd }}$ International ISRU Field Test occurred July 10-23, 2012 high up on the volcanic peak of Mauna Kea, Hawaii. This analog mission is a collaboration between NASA and its partners, primarily the Canadian Space Agency (CSA) with help from the University of Hawaii and the Pacific International Space Center for Exploration Systems (PISCES). The primary objective of the ISRU 2012 mission was to demonstrate the integrated mobility platform and the science payload for a lunar polar ice or volatile mission. To reach this objective the Regolith and Environment Science and Oxygen and Lunar Volatile Extraction (RESOLVE), mounted on the Canadian Space Agency (CSA) Artemis Jr. rover, had to demonstrate the ability to locate, characterize, and map water, ice, and other volatiles akin to what may be present at the lunar poles.

In-situ resource utilization is an important strategy for minimizing mass that needs to be launched from Earth in order to support planetary body exploration. For every 1 kilo of useful mass that could be produced on the Moon, for instance, multiple kilos of mass don't have to be launched from Earth. Being able to do this effectively to create raw materials we know are needed (e.g., oxygen) pays many times over in launch and cost reductions.

From Apollo samples, Lunar Reconnaisance Orbiter (LRO) data, Lunar Crater Observation \& Sensing Satellite (LCROSS) data, etc., we have an idea of where volatiles can be found on the Moon. In particular, there are indications that there may be a significant amount of water ice in particular locations. But the question remains to be proven by direct measurements: is this a resourse that is concentrated enough that we can reliably and effectively use it? The current concept is that a robotic precursor mission would land on the Moon, traverse the areas that showed high promise from remote sensing data and validate whether or not there really was a reliable quantity of ice in those locations. If so, ultimately large scale operations to process soil and recover the volatiles and chemicals of interest would be planned.

The mission was designed to address the following objectives: 
1. Demonstrate the functionality of an integrated rover/science payload for a lunar polar ice/volatile mission

2. Demonstrate science instruments and operations associated with performing terrain and resource site characterization before crews arrive

3. Demonstrate mission support and operations for a Lunar Robotic Precursor mission

ISRU Infrastructure: The ISRU hardware can be thought of as a rover with a highly integrated payload package that can analyze, sample, and process the soil and surrounding geology. Artemis Jr., provided by the Canadian Space Agency (CSA), is the rover (Fig. 26, 27), and carries an integrated science package known as RESOLVE. RESOLVE is designed to find, characterize and map the presence of ice and other volatiles on the lunar surface.

As Aretemis $J r$. roves from one sample location to another, small core samples are extracted from the regolith for the RESOLVE instruments to process and analyze. The RESOLVE package consists of:

- A neutron spectrometer and near-infrared spectrometer (NIR) to help locate volatiles and identify the minerals in the regolith, such as hydrogen and water vapor.

- A one-meter drill system to enable it to take samples from below the lunar surface for evaluation. These samples will be divided into smaller pieces for multiple tests.

- An oven used to bake the samples, separating the elements of interest from the soil itself.

- A volatile characterization mass spectrometer/gas chromatograph to identify the type and amount of volatiles present.

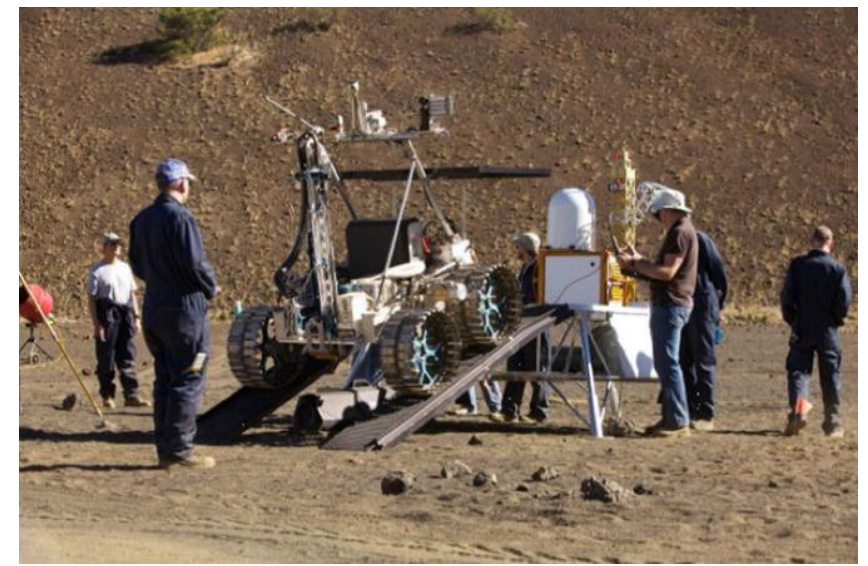

Figure 26. Simulated lander ingress/egress by Artemis Jr. rover

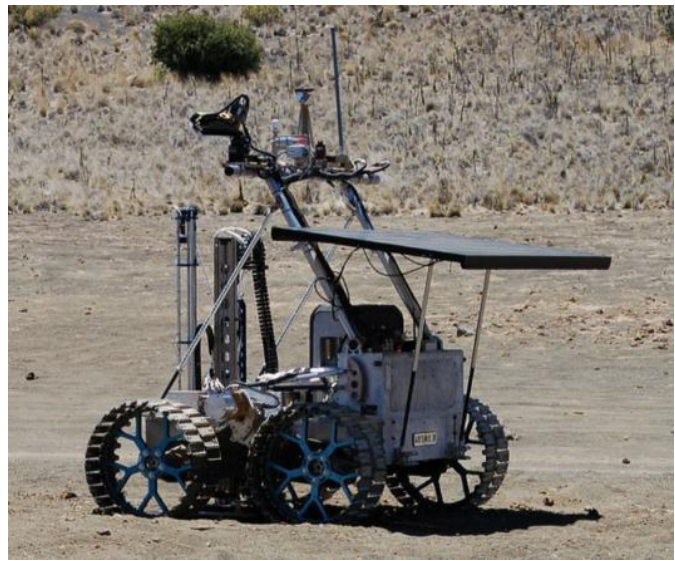

Figure 27. Resolve drill operations on Artemis $J$ r. rover

The simulation also included a mockup of a lunar lander, complete with a camera, communication, and navigation package ${ }^{15}$.

ISRU 2012 Mission: ISRU 2012 tests were conducted at Pu'u hawaihini test site which is at 9,000 ft, and also the Apollo Valley test site, which is at $11,500 \mathrm{ft}$ on Mauna Kea mountain. These locations were chosen because Hawaii's volcanic ash deposits provide geologic terrain and composition similar to what scientists expect to find on the moon, an asteroid, or Mars. The diverse terrain and rock distribution also present the same sort of mobility challenges that rovers on the moon would face ${ }^{15}$.

Being a test of precursor robotic hardware, there was not an associated crew. Also, this fundamentally differed from the other analogs described in this paper in that it represents a mature concept and hardware capability.

RESOLVE Lunar Polar Mission: Prior to operations, target sites were identified and their coordinates noted. Target sites (known as "hot spots") were prepared such that they would give the proper signatures of a location with high water content. The preparation was done in two ways: burying drill tubes with prepared soil and a known amount of water added, and also by buring small polyethylene targets around the drill tube sites (which provided the hydrogen signature that would accompany the presence of water.) The actual locations of these target sites were largely consistent with - but not always identical to - target locations in the "precursor" data (representative of remote sensing data) with which the science team was provided. The science team had no knowledge of the actual locations that had been prepared. 
Using the precursor data, the science team was able to design an optimal traverse plan for the rover. Then, telerobotically operating the rover they could traverse from one suspected site to the next, sampling at each destination. By design, as the rover approaches a location with high water or volatile content, it should detect that first with its neutron and near infrared spectrometers. That data can help guide it to the position where the peak signature is received, and the drill can then commence to take a core sample. This sample is then heated in the oven (which drives off the volatiles) and analyzed. This analysis should determine constituents and quantities of the volatiles extracted.

The RESOLVE test was highly successful, with all pieces of equipment ultimately functioning successfully in the rugged operational environment of Mauna Kea. Artemis Jr. was successfully tele-operated over a traverse distance exceeding 1 $\mathrm{km}$. The traverse can be seen in Fig. 28. A high percentage $(75 \%)$ of "hot spots" were identified, including one that hadn't shown up in the precursor data. Multiple drill operations to collect soil samples and multiple oven cycles to characterize the volatiles were also successfully

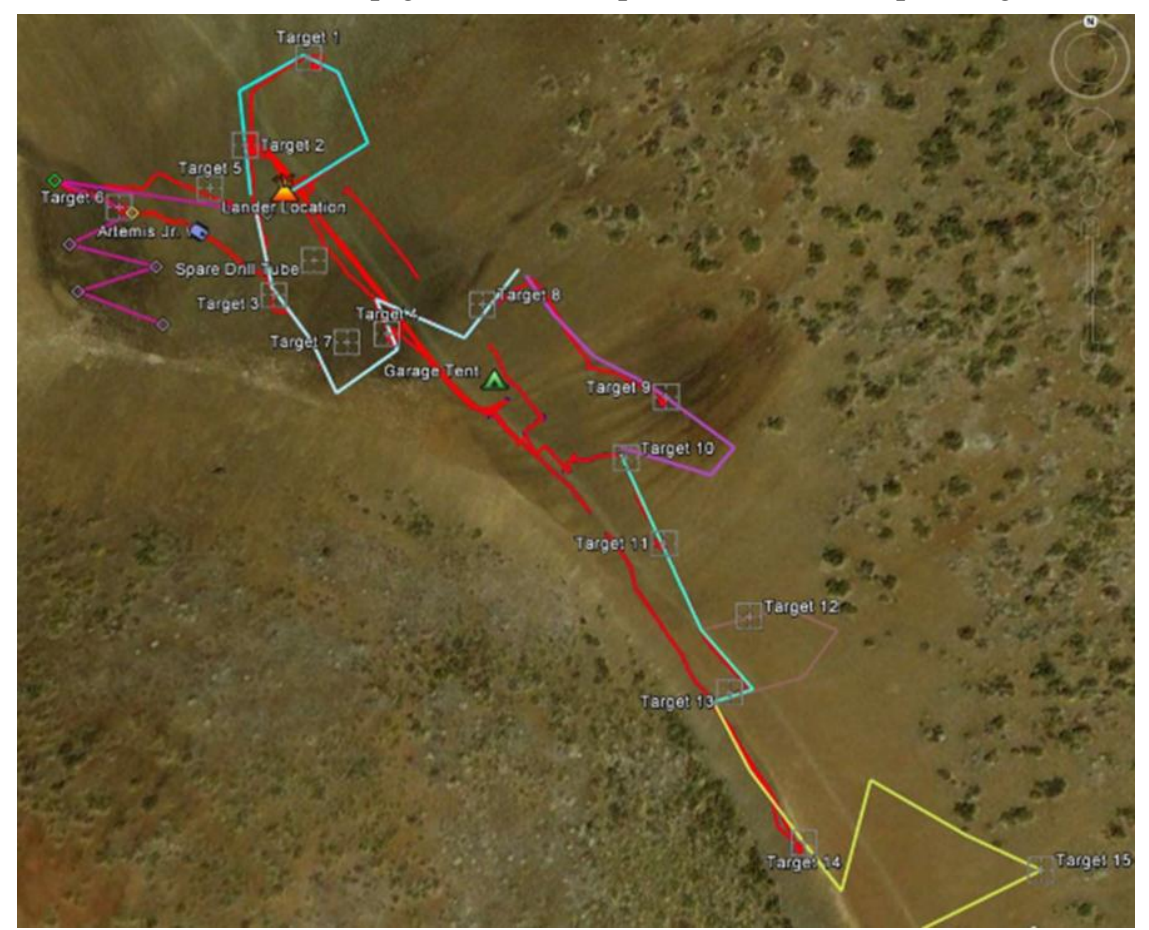

Figure 28. Complete RESOLVE mission traverse. performed.

MMAMA Science/Resource Prospecting Mission: Another suite of experiments that occurred during the ISRU test was the Moon Mars Analog Mission Activities (MMAMA). MMAMA is a group of small projects and tests that will help NASA understand how to perform new exploration techniques on the surface of the moon or Mars. These projects define the requirements for navigation, mobility, communications, sample processing and curating and other critical mission elements that could be used in future exploration missions. Some of the instruments that are part the MMAMA suite of tests includes were carried on a second rover, Juno II, also provided by CSA (Fig. 29).

The MMAMA experiments support the goal of testing potential science operations architecture that would provide geologic history and context to support rapid resource location and characterization. Geologic history is used to understand when certain conditions were prevalent, and when they ceased to exist. Geologic context is used to mean where at the site

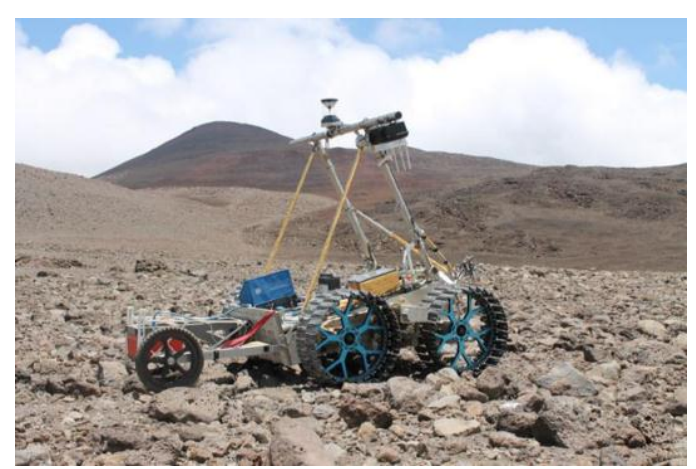

Figure 29. Juno II rover with MMAMA instruments traversing in Apollo Valley conditions were favorable for resource deposition (as recorded in the rock record).

As the rover traversed Apollo Valley, a Ground Penetrating Radar (GPR) and Mössbauer spectrometer were used to collect data that adds to the understanding of the surface and subsurface structure of the valley. The science team will continue science analysis, mining data in order to produce a geomorphic map of Apollo Valley. This will enable a comparison with results generated through traditional terrestrial field methods, and help us to better understand how to use rover-generated data to create such maps. Samples were also collected for analysis throughout its mission.

The MMAMA Science/Resource Prospecting Mission was also highly successful. The data acquisition plan was exceeded by 2-3 times, and confirmed GPR accuracy by repeating one traverse. 


\section{Conclusion}

Though the 2012 season for analog missions is still in progress, we have collected a wealth of data so far. The RATS mission in August will provide data that, in combination with data from NEEMO 16 as well as previous Desert RATS and NEEMO missions, will give us a very complete picture of the architectural elements required, operational concepts, and special tools and techniques needed to explore a Near Earth Asteroid. The preliminary results have already led us to discard some approaches as not feasible, which in turn spawned new ideas and allowed us to focus efforts on further refining the most feasible approaches.

In addition, it gives us a richer base of experience in performing human operations with a significant ( 50 second one-way) communications delay. While some mitigation is possible by changing communications techniques and expectations, it also became clear that customized tools need to be developed to truly keep the Mission Control Center and crew in synch when off nominal situations occur. Furthermore, as Just in Time Training will be a fact of life and the comm delay will make clarification difficult, it is obvious that we need to learn how to quickly develop rock solid products for that purpose.

Some initial communications delay and crew autonomy tests have already been conducted with ISTAR as well. Planning is currently underway to use the long duration and micro-gravity environment offered on ISS to simulate multiple features of a future Mars mission. Results from the ground-based analogs will be incorporated into these simulations.

The ISRU mission was a great success in several areas. First, it demonstrated hardware robustness in a challenging operational environment. Second, it allowed us to verify that we have a solid operational concept for operating the multiple instruments and rovers involved. Finally, we were able to collect more data than we had anticipated going in to the test. The ISRU hardware is now one step closer to the thermal and vacuum chamber tests that are needed to ultimately certify it for spaceflight, targeting for 2017. 


\title{
Appendix A \\ Acronym List
}

\author{
AES Advanced Exploration Systems Division (or Project) (HEOMD) \\ APS Astronaut Positioning System \\ AMCC Analog Mission Control Center \\ ARGOS Active Response Gravity Offload System \\ AU \\ Astronomical Unit \\ CSA Canadian Space Agency \\ DRM Design Reference Mission \\ DSH Deep Space Habitat \\ EAMD Exploration Analogs Mission Design \\ ESA European Space Agency \\ EV Extra-vehicular \\ EVA Extravehicular Activity \\ g Gravity \\ Gen Generation \\ GN\&C Guidance, Navigation and Control \\ GPR Ground Penetrating Radar \\ HITL Human-in-the-Loop \\ HMP Haughton Mars Project \\ HRP Human Research Program (NASA JSC) \\ ISRU In-Situ Resource Utilization \\ ISS International Space Station \\ ISTAR ISS Testbed for Analog Research \\ IVA Intra-Vehicular Activity \\ IV Intra-Vehicular (crewmember) \\ JAXA Japanese Aerospace Exploration Agency \\ JITT Just in Time Training \\ JSC Johnson Space Center (NASA) \\ LCROSS Lunar Crater Observation \& Sensing Satellite \\ LEO Low-Earth Orbit \\ LRO Lunar Recon Orbiter \\ LSB Life Support Buoy \\ $\mathrm{MeSH} \quad$ Mechanized Sample Processing and Handling System \\ MIMOS Miniaturized Mossbauer Spectrometer \\ MMAMA Moon Mars Analog Mission Activity \\ MMCC Mobile Mission Control Center \\ MMSEV Multi-Mission Space Exploration Vehicle \\ MPCV Multi-Purpose Crew Vehicle \\ MV Motor Vessel \\ NASA National Aeronautics and Space Administration \\ NEA Near Earth Asteroid \\ NEEMO NASA Extreme Environment Mission Operations \\ NIR Near-Infrared \\ NOAA National Oceanic and Atmospheric Administration
}

17

American Institute of Aeronautics and Astronautics 


$\begin{array}{ll}\text { ORU } & \text { Orbital Replacement Unit } \\ \text { PAO } & \text { Public Affairs Office } \\ \text { PISCES } & \text { Pacific International Space Center for Exploration Systems } \\ \text { PLRP } & \text { Pavilion Lake Research Project } \\ \text { RATS } & \text { Research and Technology Studies } \\ \text { RCS } & \text { Reaction Control System } \\ \text { RESOLVE } & \text { Regolith and Environment Science and Oxygen and Lunar Volatile Extraction } \\ \text { VAPoR } & \text { Volatile Analysis by Pryolysis of Regolith } \\ \text { VR } & \text { Virtual Reality }\end{array}$

\section{Acknowledgments}

This research was led by the NASA Lyndon B. Johnson Space Center, with support from other NASA centers, industry, and academia, as part of the duties of the Analog Project Management Office under the Advanced Exploration Systems.

\section{References}

1 “Analog Missions and Field Tests," National Aeronautics and Space Administration, NASAfacts NF-2011-04-534-HQ.

${ }^{2}$ Lim, D. S. S., Brady, A. L., Abercromby, A.F., Andersen, D., et al. 2011. A Historical Overview of the Pavilion Lake Research Project - Analog Science and Exploration in an Underwater Envi-ronment. in Garry, W.B., and Bleacher, J.E., eds., Analogs for Planetary Exploration: Geological Society of America Special Paper 483, p. 85-115.

3 Abercromby, A.F.J., Chappell, S.P., Gernhardt, M.L. "Desert RATS 2011: Human Exploration of Near-Earth Asteroids", Global Space Exploration Conference, Washington, DC, 22 - 24 May, 2012.

${ }^{4}$ Abell et al., 2009 "Scientific exploration of near-Earth objects via the Orion Crew Exploration Vehicle", Meteoritics and Planetary Science, 44, 1825-1836

${ }^{5}$ Abe, S., Mukai, T., Hirata, N., Barnouin-Jha, O. S., Cheng, A. F., Demura, H., Gaskell, R. W., et al. (2006). "Mass and Local Topography Measurements of Itokawa by Hayabusa". Science, 312(5778), 1344 -1347. doi:10.1126/science.1126272

${ }^{6}$ Gaffey et al., 2003 "Asteroid spectroscopy: Progress and perspectives", Meteoritics, 28, 161-187.

7 Shoemaker E. M. (1983) "Asteroid and comet bombardment of the Earth". Annual Review of Earth and Planetary Sciences, 11, 461-494.

${ }^{8}$ Mainzer et al., 2011 "NEOWISE Observations of near-Earth objects: Preliminary results", The Astrophysical Journal, 743 , 156-172.

${ }^{9}$ Abercromby, A.F.J., Gernhardt, M.L., Litaker, H. "Desert Research and Technology Studies (DRATS) 2008: Evaluation of Small Pressurized Rover and Unpressurized Rover Prototype Vehicles in a Lunar Analog Environment", NASA Technical Report 216136, 2010.

${ }^{10}$ Abercromby, A.F.J., Gernhardt, M.L., Litaker, H. "Desert Research and Technology Studies (DRATS) 2009: A 14-Day Evaluation of the Space Exploration Vehicle Prototype in a Lunar Analog Environment", NASA Technical Report (in press).

${ }^{11}$ Chappell, S.P., Abercromby, A.F.J., Gernhardt, M.L. "NEEMO 15: Evaluation of Human Exploration Systems for NearEarth Asteroids", Global Space Exploration Conference, Washington, DC, 22 - 24 May, 2012.

${ }^{12}$ Abercromby, A.F.J., Gernhardt, M.L., Jadwick, J. "Evaluation of dual multi-mission space exploration vehicle operations during simulated planetary surface exploration", Acta Astronautica (2012), doi:10.1016/ j.actaastro.2012.02.022

13 "2011 NASA Strategic Plan," National Aeronautics and Space Administration Headquarters, NP-2011-01-699-HQ, Washington DC, Feb. 2011, p. 5.

${ }^{14}$ Lee, Y.H., Eagles, D.E., Moreno, F., Rodriggs, M.A., Beisert, S.C., Stapleton, D.: "The International Space Station: Unique In-Space Test Bed as an Exploration Analog”, SpaceOps 2012 Conference, Stockholm, Sweden, June 11-15, 2012

15 “In-Situ Resource Utilization Mission,” National Aeronautics and Space Administration, NASAfacts FS-2012-07-026-JSC. 